\title{
Proton Pump Inhibitors are Risk Factors for Viral Infections: Even for COVID-19?
}

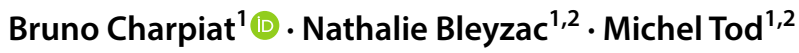

Published online: 10 August 2020

(c) Springer Nature Switzerland AG 2020

\begin{abstract}
During the ongoing pandemic of severe acute respiratory syndrome coronavirus 2 (SARS-CoV-2), more attention should be paid to the balance of risks and benefits associated with proton pump inhibitors for the following reasons. One of the main functions of gastric juice is to inactivate swallowed microorganisms, thereby inhibiting infectious agents from reaching the intestine. Studies have documented that proton pump inhibitors are a risk factor for rotavirus, influenza virus, norovirus, and Middle East respiratory syndrome coronavirus infections, and are associated with an increased risk of acute gastroenteritis during periods of highest circulation of enteric viruses. In light of the evidence for gastrointestinal infection implying a fecal-oral transmission of SARS-CoV-2 and given the magnitude of the SARS-CoV-2/coronavirus disease 2019 pandemic, associated with the widespread misuse of proton pump inhibitors, this suggests that we should not rule out the hypothesis that patients treated with proton pump inhibitors may be more at risk of being infected by SARS-CoV-2.
\end{abstract}

\section{Key Points}

Studies have documented that proton pump inhibitors are a risk factor for rotavirus, influenza virus, norovirus, and Middle East respiratory coronavirus infections, and are associated with an increased risk of acute gastroenteritis during periods of highest circulation of enteric viruses.

Regarding severe acute respiratory syndrome coronavirus 2 (SARS-CoV-2), there is evidence for gastrointestinal infection and a fecal-oral transmission.

Given the wide misuse of proton pump inhibitors and the magnitude of the SARS-CoV-2/coronavirus disease 2019 pandemic, this is a serious invitation, once more, to discontinue proton pump inhibitors in patients who do not really need them.
Bruno Charpiat

bruno.charpiat@chu-lyon.fr

1 Service Pharmacie, Hôpital de La Croix-Rousse, Groupement Hospitalier Nord, Hospices Civils de Lyon, 103 Grande Rue de la Croix-Rousse, 69004 Lyon, France

2 EMR 3738, Ciblage, Thérapeutique en Oncologie, Faculté de Médecine et de Maïeutique Lyon-Sud Charles Mérieux, Université Claude Bernard Lyon 1, Oullins Cedex, France

\section{Introduction}

During the ongoing pandemic of the severe acute respiratory syndrome coronavirus 2 (SARS-CoV-2), there is a growing interest in how exposure to certain medicines affects the risk of SARS-CoV-2 infection [1]. We suggest that more attention should be paid to the balance of risks and benefits associated with proton pump inhibitors (PPIs) for the following reasons. Proton pump inhibitors are among the top ten most widely used drugs in the world [2]. In less than 30 years, PPIs have gone from miracle drug to a major healthcare epidemic for two reasons; they are prescribed without a clear indication in up to $70 \%$ of cases, and long-term use of PPIs exposes patients to potential side effects that contribute to significant negative impacts at a population level [2].

\section{Gastric Acid, Proton Pump Inhibitors, and Viral Infections}

One of the main functions of gastric juice is to inactivate swallowed microorganisms, thereby inhibiting infectious agents from reaching the intestine. Gastric juice is thus the first line of defence against pathogens, yet very few studies have documented the effect of PPI-induced reduced gastric acidity on the susceptibility to viral infections [3]. Among those who have studied this, Weiss and Clark determined 
the sensitivity of several rotavirus strains (one bovine, two simian, and one human) to the inactivation by the natural human gastric fluids, and reported rapid inactivation of rotaviruses; each strain was inactivated at $\mathrm{pH} 2$ (half-life of $60 \mathrm{~s}$ or less) but there was only a minimal effect at $\mathrm{pH} 4$ [4]. Furthermore, Hayase et al. reported a correlation between influenza RNA positivity in gastric mucosa and acid suppression therapy, indicating that low gastric acidity may be one of the factors predisposing to influenza virus infection of the stomach [5]. Since the publication in this journal of the narrative review by Fisher and Fisher related to acidsuppressive therapy and the risk of infections [6] citing these two articles [4, 5], important information has emerged. Proton pump inhibitors appear also to be a risk factor for norovirus infection; in a retrospective case-control study, including 192 hospitalized patients positive for norovirus, Prag et al. report that there was a significantly increased risk of norovirus infection in patients treated with PPIs compared with those without (odds ratio 1.73; $95 \%$ confidence interval $1.07-2.81 ; p=0.02$ ) [7]. In addition, more recently, Vilcu et al. measured the association between continuous PPI therapy and the occurrence of acute gastroenteritis using a large French community pharmacy drug dispensation database; they found a significant association between PPI use and acute gastroenteritis (relative risk 1.81; $95 \%$ confidence interval 1.72-1.90), translating to a number needed to harm of 153 patients [8].

Regarding Middle East respiratory syndrome coronavirus (MERS-CoV), Zhou et al. demonstrated in vitro that human primary intestinal epithelial cells were highly susceptible to MERS-CoV and can sustain robust viral replication [9]. They then performed experiments in vivo in human dipeptidyl peptidase 4 transgenic mice; first, nine female mice were inoculated with 105 plaque-forming units of MERS$\mathrm{CoV}$ via intragastric gavage; three of them were pre-treated with pantoprazole to improve the viability of MERS-CoV in the mouse stomach because the in vitro experiment suggested the acid lability of MERS-CoV. The pantoprazoletreated mice sacrificed at day 5 displayed more extensive and more prominent pathology in the small intestine than the phosphate-buffered saline-treated mice. The viral load increased in the small intestine of intragastrically injected mice, especially in those pre-treated with pantoprazole [9]. In the second experiment, after direct intragastric inoculation of MERS-CoV, histological examination found MERS$\mathrm{CoV}$ enteric infection in all 12 inoculated mice, as shown by the presence of virus-positive cells, progressive inflammation, and epithelial degeneration in the small intestine, which were exaggerated in the six mice pre-treated with pantoprazole. With the progression of the enteric infection, inflammation, virus-positive cells, and live viruses emerged in the lung tissues, indicating the development of sequential respiratory infection [9].
Regarding SARS-CoV-2, Xiao et al. provided evidence for gastrointestinal infection and demonstrated that SARSCoV-2 infects gastrointestinal glandular epithelial cells [10]. Viral RNA was found in rectal swabs, even after nasopharyngeal testing has turned negative, implying a fecal-oral transmission [10], and it is of note that patients with coronavirus disease 2019 (COVID-19) who experience gastrointestinal symptoms are more likely to have severe pneumonia [11].

\section{Discussion}

Given the magnitude of the SARS-CoV-2/COVID-19 pandemic, associated with the widespread misuse of PPIs, this suggests that we cannot/should not rule out the hypothesis that patients treated with PPIs may be more at risk of being infected by COVID-19. Our hypothesis must be interpreted with caution because of the uncertainties about the causal relationship. Doubt will probably be removed by real-life studies that are sure to emerge based on dedicated COVID-19 patient registries. Luxenburger et al.'s work is the first to provide data in support of our hypothesis, as patients with COVID-19 treated regularly with PPIs before hospitalization were found to have a significantly higher mortality index [12]. They suggest that regular PPI treatment before hospital admission may be a negative predictive factor for the development of secondary infections and consecutive acute respiratory distress syndrome, and argue that it has been shown that PPIs may trigger the development of pneumonia due to the reduced gastric acid production with subsequent bacterial overgrowth in the upper gastrointestinal tract and microaspiration with following colonization of the pneumonia. They, therefore, hypothesized that PPI treatment may also be a potential risk factor for the development of secondary infections and of acute respiratory distress syndrome in hospitalized patients with COVID-19 [12]. Surprisingly, they did not refer to studies that have documented the effect of PPI-induced reduced gastric acidity on the susceptibility to viral infections [4, 5, 7-9].

The hypothesis that we put forward is, however, the opposite of that supported by Taştemur et al. who suggest that PPIs may be used for both prophylaxis and treatment; the authors base much of their reasoning on the fact that hydroxychloroquine and azithromycin, two weak bases, prevent viral spread to cells by accumulating in organelles of acidic content, raising their $\mathrm{pH}$ [13]. They concluded that PPIs show similar effects on viral entry and intracellular distribution with their effect on $\mathrm{pH}$, such as hydroxychloroquine and azithromycin [13], but emerging information questions the benefit of these drugs for preventing/treating this infection [14-16]. 


\section{Conclusions}

Pending the publication of ongoing studies in COVID-19, doubt should therefore benefit the patient. Asking patients about all medications taken, including over-the-counter drugs, and understanding why a patient is using a PPI are imperative to identifying deprescribing needs. For patients with a documented indication of PPI use, clinicians should ensure that the expected benefits are balanced against the risks of PPI therapy, and that the lowest effective dose is used for the shortest recommended duration [17].

Acknowledgements The authors thank Philip Robinson (DRCI, Hospices Civils de Lyon) for help in manuscript preparation.

Author Contributions BC performed the literature search, wrote the draft manuscript, and approved the final version. NB and MT critically examined the published data, edited the draft manuscript, and approved the final version.

\section{Declarations}

Funding This work was not supported by specific funds. It was conducted as part of our routine activity that is funded by Hospices Civils de Lyon and the University of Lyon.

Conflict of interest Bruno Charpiat, Nathalie Bleyzac, and Michel Tod have no conflicts of interest that are directly relevant to the content of this article.

\section{References}

1. Pottegård A, Kurz X, Moore N, Christiansen CF, Klungel O. Considerations for pharmacoepidemiological analyses in the SARSCoV-2 pandemic. Pharmacoepidemiol Drug Saf. 2020. https://doi. org/10.1002/pds.5029.

2. Marks D. Time to halt the overprescribing of proton pump inhibitors. Clin Pharm. 2016. https://doi.org/10.1211/CP.2016.20201 548. Available from: https://www.pharmaceutical-journ al.com/20201548.fullarticle. Accessed 9 Aug 2020.

3. Martinsen TC, Fossmark R, Waldum HL. The phylogeny and biological function of gastric juice-microbiological consequences of removing gastric acid. Int J Mol Sci. 2019;20:6031. https://doi. org/10.3390/ijms20236031.

4. Weiss C, Clark HF. Rapid inactivation of rotaviruses by exposure to acid buffer and acidic gastic juice. J Gen Virol. 1985;66:2725-30.
5. Hayase Y, Tobita K, Sato H. Detection of type B influenza virus genes from biopsied gastric mucosa. J Gastroenterol. 2002;37:101-5.

6. Fisher L, Fisher A. Acid-suppressive therapy and risk of infections: pros and cons. Clin Drug Investig. 2017;37:587-624. https ://doi.org/10.1007/s40261-017-0519-y.

7. Prag C, Prag M, Fredlund H. Proton pump inhibitors as a risk factor for norovirus infection. Epidemiol Infect. 2017;145:1617-23.

8. Vilcu AM, Sabatte L, Blanchon T, Souty C, Maravic M, Lemaitre $\mathrm{M}$, et al. Association between acute gastroenteritis and continuous use of proton pump inhibitors during winter periods of highest circulation of enteric viruses. JAMA Netw Open. 2019;2(11):e1916205. https://doi.org/10.1001/jamanetworkopen .2019 .16205 .

9. Zhou J, Li C, Zhao G, Chu H, Wang D, Hoi-Ning Yan H, et al. Human intestinal tract serves as an alternative infection route for Middle East respiratory syndrome coronavirus. Sci Adv. 2017;3(11):eaao4966.

10. Xiao F, Tang M, Zheng X, Liu Y, Li X, Shan H. Evidence for gastrointestinal infection of SARS-CoV-2. Gastroenterology. 2020;158:1831-3.

11. Zhang H, Liao YS, Gong J, Liu J, Xia X, Zhang H. Clinical characteristics of coronavirus disease (COVID-19) patients with gastrointestinal symptoms: a report of 164 cases. Dig Liver Dis. 2020. https://doi.org/10.1016/j.dld.2020.04.034.

12. Luxenburger H, Sturm L, Biever P, Rieg S, Duerschmied D, Schultheiss M, et al. Treatment with proton pump inhibitors increases the risk of secondary infections and ARDS in hospitalized patients with COVID-19: coincidence or underestimated risk factor? J Intern Med. 2020. https://doi.org/10.1111/joim.13121.

13. Taştemur Ş, Ataseven H. Is it possible to use proton pump inhibitors in COVID-19 treatment and prophylaxis? Med Hypotheses. 2020;143:110018. https://doi.org/10.1016/j.mehy.2020.110018.

14. Maisonnasse P, Guedj J, Contreras V, Behillil S, Solas C, Marlin $\mathrm{R}$, et al. Hydroxychloroquine use against SARS-CoV-2 infection in non-human primates. Nature. 2020. https://doi.org/10.1038/ s41586-020-2558-4.

15. Das S, Bhowmick S, Tiwari S, Sen S. An updated systematic review of the therapeutic role of hydroxychloroquine in coronavirus disease-19 (COVID-19). Clin Drug Investig. 2020;40:591-601.

16. Cavalcanti AB, Zampieri FG, Rosa RG, Azevedo LCP, Veiga VC, Avezum A, Coalition Covid-19 Brazil I Investigators, et al. Hydroxychloroquine with or without azithromycin in mild-tomoderate Covid-19. N Engl J Med. 2020. https://doi.org/10.1056/ NEJMoa2019014.

17. Hayes KN, Nakhla NR, Tadrous M. Further evidence to monitor long-term proton pump inhibitor use. JAMA Netw Open. 2019;2(11):e1916184. 